\title{
Promoción del buen trato y prevención del maltrato en la infancia en el ámbito de la Atención Primaria
}

\author{
F]. Soriano Faura ${ }^{a}$ y Grupo PrevInfad/PAPPS Infancia y Adolescencia ${ }^{b}$

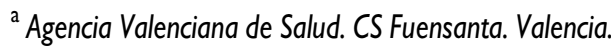

Rev Pediatr Aten Primaria. 2009; 1 I:1 2 I-44

Francisco Javier Soriano Faura, jasofa@ono.com

\begin{abstract}
Resumen
Se considera el maltrato infantil como toda acción, omisión o trato negligente, no accidental, que priva al niño de sus derechos y su bienestar, que amenaza o interfiere en su desarrollo físico, psíquico o social y cuyos autores son personas del ámbito familiar. La incidencia de maltrato en el ámbito familiar entre 2001 y 2005 ha aumentado en un 146,29\%. La negligencia es el tipo de maltrato más frecuente, representa el 86,4\% del total de los maltratos, seguido del abuso emocional y posteriormente del maltrato físico. Todos los subtipos de maltrato dan lugar a diversos trastornos de la conducta emocional, social, delictiva o de la conducta sexual, consumo de drogas ilegales o ideas suicidas. Los servicios de Atención Primaria tienen un papel destacado en la prevención del maltrato infantil, al ser los únicos servicios comunitarios a los que tienen acceso normalizado y generalizado las familias, en un periodo de edad en el que el niño es especialmente vulnerable. De las actuaciones que clásicamente se recomiendan solo las actividades sobre familias de riesgo de ocurrencia de maltrato a la infancia han resultado de alguna eficacia. La actividad más consistente en prevención, con mejores resultados, es la visita domiciliaria en familias de riesgo desde la etapa perinatal a los 2-3 años de vida.
\end{abstract}

Palabras clave: Maltrato, Buen trato, Infancia, Atención Primaria, Prevención.

Abstract

Child abuse is any act, omission or neglect, not accidental, that deprives children from rights and well-being, it threatens or interferes with their physical, psychological or social development, it is committed by parents or caregivers. The incidence of abuse in the family environment has increased by 146.29\% from 2001 to 2005.

\footnotetext{
${ }^{\text {b}}$ Grupo Previnfad: Cortés Rico, Olga; Esparza Olcina, María Jesús; Galbe Sánchez-Ventura, José; Martínez Rubio Ana; Merino Moína, Manuel; Pallás Alonso, Carmen Rosa; Pericas Bosch, Juan; Sánchez Ruiz-Cabello, Francisco Javier; Soriano Faura, Francisco Javier; Colomer Revuelta, Julia.
}

Los autores declaran no presentar conflictos de intereses en relación con la preparación y publicación de este artículo. 
Neglect is the most frequent child abuse; it represents $86.4 \%$ of all maltreatments, followed by emotional and physical abuse. All subtypes of abuse produce several emotional, social, criminal or sexual behaviour impairment, illegal drug consumption or suicide ideation. Primary Care has an outstanding role in child abuse prevention because it is the only community service where families have a regular and generalized access in the period of time when children are especially vulnerable. The interventions on risk families are the only ones that have proved some degree of effectiveness. The most consistent intervention in prevention, and with the best results, is the home visit to risk families with children whose ages range from the perinatal period until 2-3 years of life.

Key words: Child abuse, Good treatment, Child, Primary Care, Prevention

Definición y tipos de maltrato infantil intrafamiliar

Una de las definiciones más claras de las hasta ahora publicadas es la que considera el maltrato infantil como toda acción, omisión o trato negligente, no accidental, que priva al niño de sus derechos y su bienestar, que amenaza o interfiere en su desarrollo físico, psíquico o social y cuyos autores son personas del ámbito familiar.

De forma sencilla y operativa se distinguen los siguientes tipos de maltrato': maltrato físico, negligencia, abuso sexual y maltrato emocional.

- Maltrato físico: toda acción voluntariamente realizada que provoque o pueda provocar lesiones.

- Negligencia: la omisión de una acción necesaria para atender el desarrollo y el bienestar físico y psicológico de un menor. La negligencia intrafamiliar viene a identificarse con el abandono de las obligaciones que socialmente son encomendadas a los padres o tutores de un menor.

- Abuso sexual: cualquier comportamiento en el que un menor es utilizado por un adulto $u$ otro menor como medio para obtener estimulación o gratificación sexual. Se incluyen el voyeurismo, exhibicionismo, tocamientos y penetración. También la inducción de un menor a la prostitución por parte un familiar, aunque la relación sexual se mantenga con terceros.

- Maltrato emocional: cualquier acción, normalmente de carácter verbal, o cualquier actitud de un adulto hacía un menor que provoquen, o puedan provocar en él daños psicológicos.

Además debemos incluir:

- Maltrato prenatal: conductas realizadas voluntariamente por la 
madre o personas del entorno familiar, o conductas negligentes que influyen negativamente en el embarazo y repercuten en el feto.

- Síndrome de Münchausen por poderes: los padres, frecuentemente la madre, provocan o inventan síntomas orgánicos o psicológicos en sus hijos que inducen a someterlos a exploraciones, tratamientos e ingresos hospitalarios innecesarios. En ocasiones pueden agravar enfermedades o síntomas preexistentes en el niño.
Magnitud del problema del maltrato infantil

- La incidencia de maltrato en el ámbito familiar entre 2001 y 2005 ha aumentado en un $146,2 \%$ (figura 1).

- Por tramos de edad, el grupo donde la incidencia de maltrato es mayor es el de los menores de 13 años (figura 1).

- La prevalencia por 1.000 niños menores de 18 años ha tenido una evolución similar, con un incremento del $133,3 \%$ del 2001 a 2005 (de 0,36 a 0,84 por 1.000 niños).

Figura 1. Incidencia del maltrato en menores de 18 años, por grupos de edad y años.

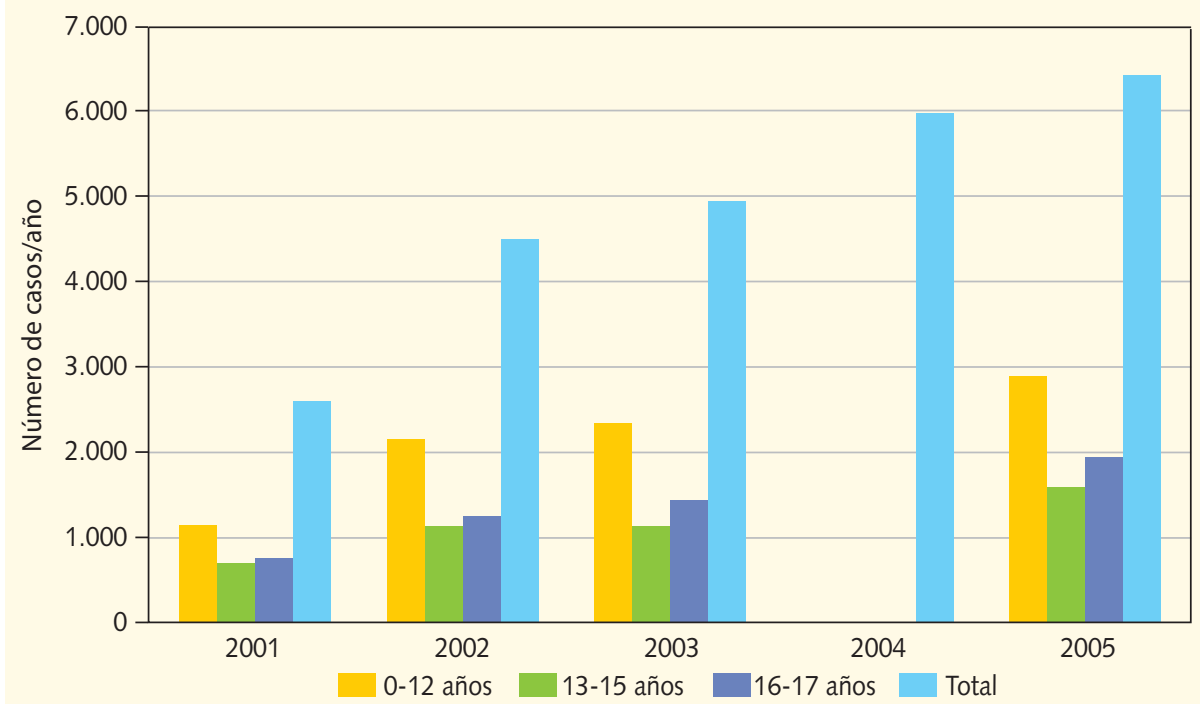

Fuente: Ministerio del Interior; Centro Reina Sofía, Valencia. 
Aunque en la bibliografía se reitera que los casos que llegan a los servicios sociales son apenas el $20 \%$ del total de sucesos de maltrato infantil, nosotros creemos que los datos del Programa Nacional de Epidemiología de la Violencia en el Ámbito Familiar tienen sufi- ciente solidez para conocer el fenómeno epidemiológico del maltrato infantil2.

La negligencia es el tipo de maltrato más frecuente, representa el $86,4 \%$ del total de los maltratos, seguido del abuso emocional y posteriormente del maltrato físico. Los casos de abuso sexual

Tabla I. Incidencia del maltrato en diferentes países

\begin{tabular}{l|l|l|l|l}
\hline & Años & $\begin{array}{l}\text { Casos } \\
\text { informados }\end{array}$ & $\begin{array}{l}\text { Casos } \\
\text { comprobados }\end{array}$ & $\begin{array}{l}\text { Frecuencia } \\
\text { por 1.000 niños }\end{array}$ \\
\hline Australia & $2002-2003$ & 198.355 & 40.416 & 6,8 \\
\hline Canadá & 1998 & 312.373 & 61.000 & 9,7 \\
\hline Reino Unido & $2002-2003$ & 570.220 & 30.200 & 2,7 \\
\hline EE. UU. & 2001 & 2.673 .000 & 492.100 & 12,4 \\
\hline España & $1997-1998$ & 32.741 & 11.148 & 0,71 \\
\hline
\end{tabular}

Fuente: Creighton S4, 2004 (modificado al incluir los datos epidemiológicos de España).

Figura 2. Prevalencia por 10.000 niños según tipo de maltrato.

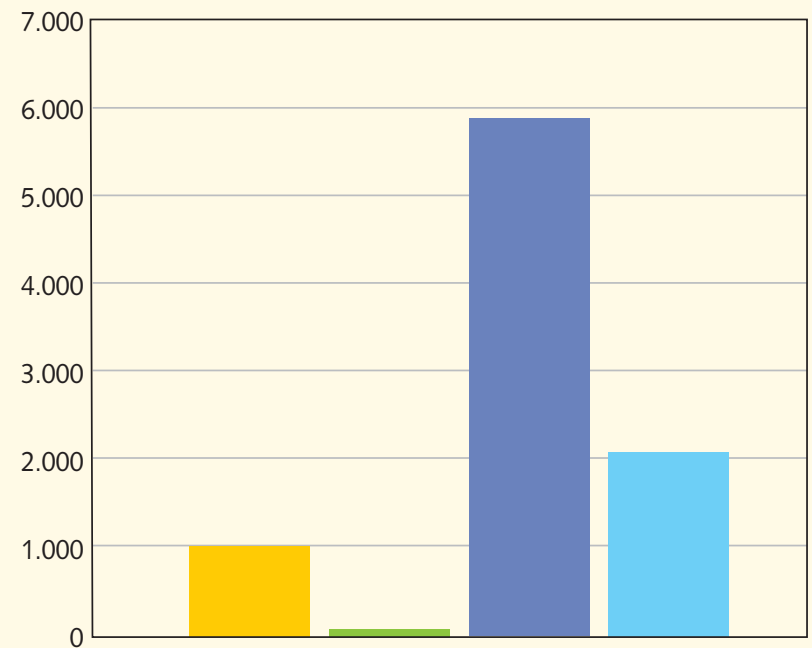

Maltrato físico

Abuso sexual

Negligencia

Maltrato emocional

Nota: una víctima puede sufrir uno o más tipos de maltrato. 
sólo representan el 3,6\% de todos los casos informados de maltrato (figura 2). La baja prevalencia del abuso sexual (0,37 por 10.000 niños) contrasta con estudios realizados en encuestas a adultos de los que el $18,9 \%$ manifestaba haber sufrido abusos sexuales en la infancia, $15 \%$ de los varones y $22 \%$ de las mujeres ${ }^{3}$.

\section{¿La prevalencia de maltrato \\ en España es comparable \\ a la de otros países?}

En la tabla I distinguimos los casos informados por las principales agencias de Canadá, Australia, Reino Unido y EE. UU. y casos confirmados después de la investigación de los mismos. En Australia, Canadá y EE. UU. se sospechan aproximadamente 5 veces más casos de los que finalmente son confirmados. La incidencia de estos 3 países es sustancialmente más alta que en Reino Unido y España, esto es proba- blemente debido a los sistemas de detección e información del maltrato y los programas de prevención y protección al menor de cada uno de estos países y en menor medida debido a las diferencias entre las poblaciones sujetas al maltrato ${ }^{4}$.

\section{¿Cuál es la relación existente entre los diferentes tipos de maltrato infantil?}

Un rasgo característico es que no se dé un solo tipo de maltrato, sino que es frecuente la combinación con otros tipos de maltrato. En el estudio de Cerezo ${ }^{5}$ sobre una muestra de 237 casos de la Comunidad Valenciana en 1997 (expedientes confirmados de maltrato infantil) se aprecia por ejemplo que de 188 niños que habían sufrido abandono físico, 48 también recibieron maltrato físico (tabla II). Por lo que respecta al abuso sexual destaca la combinación entre este y el abuso emocional.

Tabla II. Distribución de frecuencias con respecto al tipo de maltrato

\begin{tabular}{l|l|l|l|l|l}
\hline & $\begin{array}{l}\text { Abandono } \\
\text { emocional }\end{array}$ & $\begin{array}{l}\text { Abuso } \\
\text { emocional }\end{array}$ & $\begin{array}{l}\text { Abandono } \\
\text { físico }\end{array}$ & $\begin{array}{l}\text { Abuso } \\
\text { físico }\end{array}$ & $\begin{array}{l}\text { Abuso } \\
\text { sexual }\end{array}$ \\
\hline Abandono emocional & 150 & - & - & - & - \\
\hline Abuso emocional & 83 & 123 & - & - & - \\
\hline Abandono físico & 124 & 104 & 188 & - & - \\
\hline Abuso físico & 32 & 48 & 48 & 58 & - \\
\hline Abuso sexual & 10 & 13 & 11 & 10 & 15 \\
\hline
\end{tabular}


Otras publicaciones insisten igualmente en la combinación entre los diferentes tipos de maltrato; Edwars (2003) ${ }^{6}$ en los expedientes de 8.667 adultos de una institución de salud mental comunica que el $34,6 \%$ de los sujetos que habían sido sujetos a maltrato infantil recibieron más de un tipo de abuso; Scher (2004) ${ }^{7}$ en cuestionarios administrados a una muestra representativa de la ciudad de Memphis de 967 adultos encuentra que el 13\% de los participantes declaraban más de un tipo de maltrato infantil.

Otra condición del maltrato publicada en el estudio de Cerezo ${ }^{5}$ de la Comunidad Valenciana y que con toda probabilidad es característico del fenómeno del maltrato infantil en cualquier población es que el maltrato sea reiterado, $97,1 \%$ de los expedientes estudiados en la Comunidad Valenciana son de niños con abuso reiterado.

\section{Consecuencias}

Independientemente de las secuelas físicas que desencadena directamente la agresión producida por el abuso físico o sexual, todos los subtipos de maltrato dan lugar a diversos trastornos; las consecuencias más comunes ${ }^{8-10}$ se muestran en la tabla III.

Lansford y cols. ${ }^{11}$ realizaron un estudio prospectivo a lo largo de 12 años, observando que el $74 \%$ de los adoles-

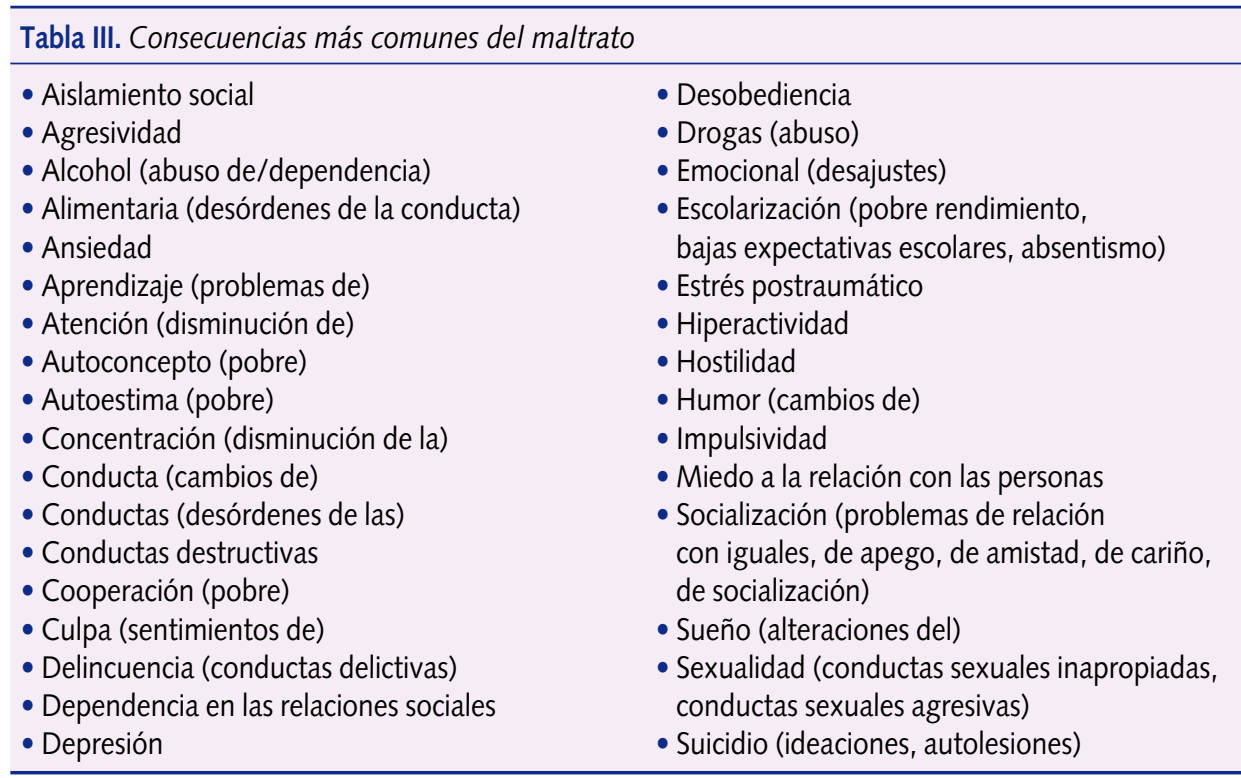


centes que habían sufrido maltrato físico presentaron algún problema de conducta frente al $43 \%$ de los chicos que no habían sufrido maltrato físico. Además presentaron más de tres problemas de conducta el $21 \%$ de los niños con maltrato frente al $7 \%$ del grupo que no recibió abuso físico. Las chicas que sufrieron abuso físico o sexual podían presentar con mayor probabilidad conductas agresivas (odds ratio [OR] 7,1), depresión (OR 7,2), ansiedad (OR 9,3) o estrés postraumático $(\mathrm{OR} 9,8)^{12}$.

En nuestro medio se ha comprobado retraso en el desarrollo psicológico en niños menores de 5 años que sufrieron abuso (Osuna, 2000) ${ }^{13}$.

Se estima que el riesgo de tentativa de suicidio en adolescentes maltratados es cinco veces más alto, comparado con aquellos que no presentaron maltrato en la infancia ${ }^{14}$.

Otra consecuencia a largo plazo es la violencia transgeneracional, un niño maltratado tiene alto riesgo de ser perpetrador de maltrato en la etapa adulta a su pareja o a sus hijos. Un metaanálisis publicado en 2000 encuentra evidencia de que existe continuidad intergeneracional del abuso infantil, el riesgo relativo de transmisión del abuso de una generación a otra es de 12,6 (intervalo de confianza [IC] $95 \%: 1,82-87,2)^{15}$.
¿Por qué un porcentaje apreciable de niños maltratados no desarrollan desórdenes de la conducta 0 alteraciones psiquiátricas?

Aproximadamente el $25-50 \%$ de los niños sometidos a maltrato grave se encontraban asintomáticos, no presentaban alteraciones emocionales o desórdenes psiquiátricos, en el momento de la valoración ${ }^{14}$. Además, es muy heterogénea la sintomatología observada a través del seguimiento de estos niños, lo que sugiere que las consecuencias son resultado de múltiples factores que interactúan originando atribuciones o ajustes capaces de favorecer conductas saludables o trastornos de la conducta y desórdenes psiquiátricos.

Es decir, que niños sometidos a condiciones de vida difíciles o traumas a veces graves consiguen desenvolverse $y$ vivir, incluso en un nivel superior, como si el trauma vivido y asumido hubiera desarrollado en ellos recursos para elaborar una respuesta de ajuste saludable a la adversidad, es esto lo que se viene a llamar resiliencia o capacidad de una persona para mantener un funcionamiento efectivo frente a las adversidades del entorno o recuperarlo en esas condiciones.

En relación al género, ser mujer es un factor predictivo positivo para te- 
ner resiliencia o mejor adaptación a sucesos de maltrato: el $26,6 \%$ de las chicas maltratadas presentaron adecuada resiliencia frente al $17,6 \%$ de los chicos. Incluso en el grupo control ser mujer es un factor protector para tener resiliencia: el 51,3\% de las mujeres frente al $32,5 \%$ de los hombres del grupo control. Por tipo de maltrato, el abuso sexual y la negligencia se muestran como predictores negativos de un buen ajuste, no se observa relación entre género y tipo de maltrato para predecir mejor o peor ajuste saludable al maltrato ${ }^{16}$.

Otros factores que de forma directa o indirecta intervienen en la resiliencia tienen relación con: intensidad, duración y/o frecuencia del maltrato; características del niño; el uso o no de la violencia física; relación más o menos directa con el agresor; apoyo intrafamiliar a la víctima infantil; y el acceso y competencia de los servicios de ayuda médica, social y psicológica.

Un factor que ha mostrado gran importancia es el relativo a las atribuciones cognitivas (consideraciones subjetivas, perceptivas, para elaborar lo que le ha sucedido) que realiza el niño acerca del abuso sufrido. El proceso de elaboración del suceso y la forma de hacer frente al abuso estan íntima- mente relacionados con la adaptación al estrés vital ${ }^{17,18}$.

\section{Factores de riesgo asociados}

al maltrato

La mayoría de los estudios efectuados para conocer los factores que explican el maltrato (factores personales, familiares y socioculturales) provienen de familias en las que han intervenido servicios de protección infantil, lo que ha llevado a un error sistemático profesional de identificación y señalamiento del abuso. Se admite que no hay características demográficas o familiares que excluyan la posibilidad de abuso en niños.

El modelo etiopatogénico que mejor explica la presencia de factores de riesgo que pueden inducir al abuso, es el modelo integral del maltrato infantil ${ }^{14}$. Este modelo considera la existencia de diferentes niveles ecológicos que están encajados unos dentro de otros interactuando en una dimensión temporal. Existen en este sistema factores compensadores (factores protectores) que actuarían según un modelo de afrontamiento, impidiendo que los factores potenciadores (factores de riesgo o de vulnerabilidad) que se producen en las familias desencadenen una respuesta agresiva hacia sus miembros. La progresiva disminución de los factores compensadores podría expli- 
car la espiral de violencia intrafamiliar que se produce en el fenómeno de maltrato infantil (tabla IV).
Wilson (1995) publica un metaanálisis en el que encuentra relación entre abuso infantil y bajo soporte social, recien-

Tabla IV. Modelo integral del maltrato infantil

\begin{tabular}{|c|c|c|}
\hline Niveles ecológicos & Factores potenciadores & Factores compensadores \\
\hline $\begin{array}{l}\text { Desarrollo individual } \\
\text { de los padres }\end{array}$ & $\begin{array}{l}\text { - Historia familiar de abuso } \\
\text { - Falta de afectividad en la infancia } \\
\text { de los padres } \\
\text { - Baja autoestima } \\
\text { - Pobres habilidades personales }\end{array}$ & $\begin{array}{l}\text { - Experiencia en los cuidados del niño } \\
\text { - Habilidad interpersonal } \\
\text { - Coeficiente intelectual elevado } \\
\text { - Reconocimiento de la experiencia } \\
\text { de maltrato en la infancia }\end{array}$ \\
\hline $\begin{array}{l}\text { Medio familiar: } \\
\text { a) Padres }\end{array}$ & $\begin{array}{l}\text { - Trastornos físicos/psíquicos (inclu- } \\
\text { ye ansiedad y depresión) } \\
\text { - Drogodependencias } \\
\text { - Padre/madre solo } \\
\text { - Madre joven } \\
\text { - Padre/madre no biológico } \\
\text { - Disarmonía familiar } \\
\text { - Enfermedades/lesiones } \\
\text { - Conflictos conyugales } \\
\text { - Violencia familiar } \\
\text { - Falta de control de impulsos }\end{array}$ & $\begin{array}{l}\text { - Planificación familiar } \\
\text { - Satisfacción personal } \\
\text { - Escasos sucesos vitales estresantes } \\
\text { - Intervenciones terapéuticas } \\
\text { en la familia } \\
\text { - Ambiente familiar sin exposición } \\
\text { a violencia } \\
\text { - Armonía marital }\end{array}$ \\
\hline $\begin{array}{l}\text { Medio familiar: } \\
\text { b) Hijos }\end{array}$ & $\begin{array}{l}\text { - Hijos no deseados } \\
\text { - Trastorno congénito } \\
\text { - Anomalías físicas o psíquicas } \\
\text { - Enfermedad crónica } \\
\text { - Tamaño de la fatria } \\
\text { - Nacimiento prematuro } \\
\text { - Bajo peso al nacer } \\
\text { - Ausencia de control prenatal }{ }^{20} \\
\text { - Trastorno de la conducta del hijo }{ }^{21} \\
\text { - Proximidad de edad entre hijos }\end{array}$ & $\begin{array}{l}\text { - Apego materno/paterno al hijo } \\
\text { - Satisfacción en el desarrollo } \\
\text { del niño }\end{array}$ \\
\hline $\begin{array}{l}\text { Exosistema: } \\
\text { a) Sociolaboral }\end{array}$ & $\begin{array}{l}\text { - Bajo nivel social/económico } \\
\text { - Desempleo } 22 \\
\text { - Insatisfacción laboral }\end{array}$ & - Condición financiera \\
\hline $\begin{array}{l}\text { Exosistema: } \\
\text { b) Vecindario }\end{array}$ & - Aislamiento social & $\begin{array}{l}\text { - Apoyo social } \\
\text { - Buena experiencia con iguales }\end{array}$ \\
\hline $\begin{array}{l}\text { Macrosistema: } \\
\text { a) Sociales }\end{array}$ & $\begin{array}{l}\text { - Alta criminalidad } \\
\text { - Baja cobertura servicios sociales } \\
\text { - Alta frecuencia desempleo } \\
\text { - Pobreza de grupo social23 } \\
\text { - Alta movilidad geográfica } \\
\end{array}$ & $\begin{array}{l}\text { - Programas de mejora, de redes } \\
\text { de apoyo e integración social } \\
\text { de familias vulnerables }{ }^{24} \\
\text { - Programas sanitarios }\end{array}$ \\
\hline $\begin{array}{l}\text { Macrosistema: } \\
\text { b) Culturales }\end{array}$ & - Aceptación castigo corporal & $\begin{array}{l}\text { - Actitud hacia la infancia, la mujer, } \\
\text { la paternidad de la sociedad }\end{array}$ \\
\hline
\end{tabular}


tes sucesos vitales estresantes, enfermedad siquiátrica en la madre, embarazo no deseado e historia de abuso en la infancia de la madre o el padre ${ }^{25}$.

\section{¿Un solo factor de riesgo explica la existencia de maltrato en el niño?}

La génesis del maltrato no se explica por la presencia de un solo factor de riesgo, se explica más bien por la existencia de una acumulación de factores de riesgo.

Brown et al. ${ }^{26}$ realizan un estudio longitudinal de 644 familias entre 1975 y 1992, observando que la prevalencia de abuso o trato negligente aumenta del $3 \%$, cuando no se encuentra ningún factor de riesgo, al $24 \%$ cuando se observan 4 o más factores de riesgo. En España, Olivan (2002) publica un estudio retrospectivo en el que observa que la causa del maltrato infantil no está relacionado aisladamente con la presencia de discapacidad infantil, sino que está relacionado con la existencia de factores de riesgo familiar y del entorno, de forma que cuantos más de estos factores se asocien alrededor del niño discapacitado mayor es la posibilidad de maltrato. El 93,5\% de los niños discapacitados que habían recibido maltrato al menos tenían otro factor de riesgo además de la discapacidad y el $64,5 \%$ presentaron más de uno ${ }^{27}$.
Estudios realizados en nuestro país ${ }^{28}$ nos indican que:

- En familias monoparentales se pone de manifiesto el papel protector del apoyo social, la probabilidad de abuso era 5,6 veces menor que en familias monoparentales sin apoyo social.

- Las dificultades económicas estaban significativamente asociadas al maltrato en familias biparentales, con un aumento de la probabilidad de 6,3 veces sobre familias biparentales sin dificultades económicas.

- El bajo nivel educativo está asociado en familias monoparentales a maltrato, siendo la probabilidad de un 28,7. En familias biparentales, el bajo nivel educativo aumentó la probabilidad del maltrato en 11,4.

- El mayor número de hijos estaba relacionado con una probabilidad mayor de ocurrencia de maltrato (razón = 4,0).

Se sabe que en el $30-70 \%$ de las familias en que se abusa de un adulto habrá abuso en menores ${ }^{29}$.

Los factores que están asociados a la co-ocurrencia de violencia doméstica y trato negligente infantil, en comparación con la existencia exclusiva de trato negligente sin que exista violencia do- 
mestica, son ${ }^{29}$ : familia monoparental $(\mathrm{OR}=6,57)$; la madre abusa de alcohol o drogas $(\mathrm{OR}=2,87)$; la madre tiene problemas psiquiátricos $(\mathrm{OR}=5,22)$; la madre es la maltratadora del niño $(O R=$ 2,56).
La co-ocurrencia entre violencia doméstica y abuso físico estuvo asociada, comparada con la existencia de abuso infantil sin datos de violencia domestica, $\operatorname{con}^{30}$ : el padre abusa de alcohol o drogas en la época en que maltrata al niño

Tabla V. Efectividad de la prevención del maltrato infantil en el ámbito de la Atención Primaria

\begin{tabular}{|c|c|c|c|}
\hline Intervención & Efectividad & Nivel de evidencia & $\begin{array}{l}\text { Fuerza de } \\
\text { recomendación }\end{array}$ \\
\hline $\begin{array}{l}\text { Identificación de familias } \\
\text { de riesgo mediante el } \\
\text { uso de cuestionarios o } \\
\text { listados }\end{array}$ & \begin{tabular}{|l|} 
Identifica \\
inadecuadamente a \\
familias, probabilidad alta \\
de falsos positivos
\end{tabular} & II-2 (estudios de cohortes) & $\mathrm{E}^{44-46}$ \\
\hline $\begin{array}{l}\text { Actividades de } \\
\text { promoción del buen } \\
\text { trato. Educación para la } \\
\text { salud, disciplina y } \\
\text { solución de conflictos a } \\
\text { familias con bajo riesgo }\end{array}$ & $\begin{array}{l}\text { No se han encontrado } \\
\text { estudios que prueben } \\
\text { que las intervenciones } \\
\text { en la población en general } \\
\text { se puedan recomendar } \\
\text { en los exámenes periódicos } \\
\text { de salud }\end{array}$ & $\begin{array}{l}\text { III (recomendado por } \\
\text { expertos) }\end{array}$ & I \\
\hline $\begin{array}{l}\text { Programas } \\
\text { multidisciplinares de } \\
\text { apoyo social, } \\
\text { psicológico y educativo } \\
\text { a padres de riesgo } \\
\text { (incluye la intervención } \\
\text { en violencia doméstica) }\end{array}$ & $\begin{array}{l}\text { Aumentan los } \\
\text { conocimientos pero no se } \\
\text { ha establecido que } \\
\text { reduzcan el abuso o el } \\
\text { abandono }\end{array}$ & I (estudios aleatorizados) & $1^{47,48}$ \\
\hline $\begin{array}{l}\text { Educación para la salud } \\
\text { en familias de riesgo }\end{array}$ & $\begin{array}{l}\text { Disminuyeron en el grupo } \\
\text { de intervención los } \\
\text { informes de abuso. } \\
\text { Aunque el estudio } \\
\text { presenta problemas de } \\
\text { diseño metodológico }\end{array}$ & $\begin{array}{l}\text { II-1 (ensayos clínicos sin } \\
\text { aleatorización) }\end{array}$ & $1^{49}$ \\
\hline $\begin{array}{l}\text { Aumento de las visitas } \\
\text { de seguimiento del } \\
\text { control de salud }\end{array}$ & $\begin{array}{l}\text { No se evidenció } \\
\text { disminución de la } \\
\text { notificación de abusos o } \\
\text { trato negligente }\end{array}$ & $\| 1$ & $1^{47}$ \\
\hline Visita domiciliaria & $\begin{array}{l}\text { Disminuyen la incidencia de } \\
\text { abuso y trato negligente, } \\
\text { además de otros resultados } \\
\text { favorables para la salud del } \\
\text { niño y la familia }\end{array}$ & 1 & \\
\hline
\end{tabular}


$(\mathrm{OR}=5,19)$; el padre tiene historial violento o ha sido arrestado en alguna ocasión por actos violentos $(O R=6,11)$.

En un estudio longitudinal de 2.544 madres que tuvieron su primer hijo y recibieron programa de atención a domicilio para la prevención del abuso infantil, se encontró una relación significativa entre la violencia doméstica y los siguientes tipos de maltrato infantil: abuso físico, maltrato emocional y negligencia, estando asociados la violencia doméstica y el maltrato en el $38 \%$ de los casos confirmados (155 niños). La violencia doméstica precedió al maltrato del niño en el $78 \%$ de los casos y fue independiente de la observación de los servicios de protección al menor ${ }^{31}$.

\section{Intervenciones preventivas \\ en el maltrato infantil}

Los servicios de Atención Primaria (AP) tienen un papel destacado en la prevención del maltrato infantil, al ser los únicos servicios comunitarios a los que tienen acceso normalizado y generalizado las familias, en un periodo de edad en el que el niño es especialmente vulnerable (menores de 5 años de edad).

Pediatras y personal de Enfermería de AP se encuentran en una posición favorable para detectar niños en situación de riesgo, colaborar en la intervención protectora de la población general y realizar actividades preventivas en niños considerados de riesgo.

La prevención se va a desarrollar principalmente en menores de 5 años, ya que a partir de esta edad es fundamentalmente la escuela la que tiene un papel principal en la actuación en el maltrato a la infancia.

\section{Niveles de actuación}

Prevención primaria ${ }^{32,33}$, dirigida la población general con el objetivo de evitar la presencia de factores estresores o de riesgo y potenciar los factores protectores:

1. Sensibilización y formación de profesionales de Atención Primaria en la detección y prevención del maltrato infantil.

2. Intervenir en foros comunitarios de educación para la salud incrementando las habilidades de los padres en el cuidado de los hijos, en las relaciones educativas y afectivas que se establecen en la relación padres-hijos:

- Cursos de preparación al parto.

- Escuelas de padres u otros centros comunitarios, promoviendo valores de estima hacia la infancia, la mujer y la paternidad.

3. Prevenir el embarazo no deseado, 
principalmente en mujeres jóvenes, mediante:

- La educación sexual en centros escolares.

- El programa de salud infantil (controles de salud de los 11, 12 y 14 años realizados en $A P)$.

- Facilitar recursos de planificación familiar.

4. Evaluar la calidad del vínculo afectivo padres-hijos, los cuidados al niño, presencia de síntomas que sugieren abandono o carencia afectiva, actitud de los padres frente al establecimiento de normas y límites (azotes, castigos o correcciones verbales desproporcionadas).

5. Intervenir en las consultas con amabilidad y empatía cuando observamos prácticas de castigo corporal que se establecen inapropiadamente en la relación padres e hijos (nalgadas, sacudidas, amenazas, reprimenda con abuso psíquico, etc.), discutiendo métodos alternativos de disciplina, refuerzos de experiencias de confrontación padres-hijo.

6. Utilizar "guías anticipadoras" para comunicar a cada edad específica los requerimientos del niño (alimentación, higiene, sueño, cólico del lactante, rabietas, control de esfínteres, etc.), reconocer la dificultad que entraña cada nuevo periodo del desarrollo, brindar orientación práctica en cuanto al esta- blecimiento de disciplina constructiva y promover la estimulación del niño y el crecimiento emocional estable.

7. Identificar los puntos valiosos y positivos de los padres, alabar sus esfuerzos, reforzar la autoestima y la competencia.

Prevención secundaria, dirigida a la población de riesgo con el objetivo de reducir daños y atenuar los factores de riesgo presentes, potenciando los factores protectores:

1. Reconocer situaciones de abandono o trato negligente en el niño. Evaluar la situación de negligencia y consultar con los servicios de Protección al Menor.

2. Reconocer situaciones de violencia doméstica o de abuso a la mujer como una medida efectiva de prevenir el maltrato infantil (30-70\% de las familias en que se abusa de un adulto habrá abuso en menores) $)^{34,35}$.

3. Remitir a los miembros de la familia a un centro de terapia psicológica para educar en el "manejo del enfado y la ira"36-39.

4. Remitir a centros de salud mental a padres con adicción a alcohol, drogas o trastornos psiquiátricos. Recomendar el tratamiento por su médico de familia de los trastornos de ansiedad o depre$\operatorname{sivos}^{32}$. 
5. Ofrecer a las familias que lo precisen todos los recursos comunitarios de ayuda psicológica a adultos y niños, social, laboral o económica ${ }^{33}$.

6. Colaborar con el trabajador social de la zona en la definición de objetivos, planes, estrategias y ayudas definidas para cada familia de riesgo.

7. Visita domiciliaria realizada por Enfermería a familias de alto riesgo, desde la etapa prenatal hasta los 2 años de vi$\mathrm{da}^{40-42}$.

8. Aumentar las visitas dentro del programa de salud infanti ${ }^{43}$.

\section{Efectividad de la prevención del maltrato infantil desde Atención Primaria}

No existe consenso sobre las intervenciones preventivas en el abuso infantil (tabla V). En parte es debido a que el estudio de la prevención del maltrato está limitada por la complejidad del problema, la dificultad de medir las variables e interpretar los resultados, el desconocimiento de cómo interactúan las variables que determinan el riesgo y el consiguiente abuso. Además, es notable la diferencia de programas que sostienen estas intervenciones, diferentes en sus niveles de actuación, sus recursos humanos y económicos, sostenidos por pequeñas evidencias que justifiquen la efectividad de estos programas.

¿Es efectivo el uso de instrumentos de cribado (cuestionarios, listas de riesgos) en los centros de salud, para identificar familias con riesgo de abuso infantil?

La identificación de familias de riesgo e igualmente de la violencia doméstica parece parte importante de las estrategias de promoción del buen trato y prevención del maltrato en la infancia en el sector sanitario, la cuestión es si existen instrumentos de cribado o métodos de detección eficaces para señalar o identificar familias con riesgo potencial de abusar de sus hijos.

La US Preventive Services Task Force (USPSTF) ${ }^{44}$ realizó una revisión sistemática de la literatura existente entre 1982 y 2002, observando que los cuestionarios autoadministrados por los padres o los realizados por los sanitarios no eran útiles para identificar a la población de riesgo a pesar de su alta sensibilidad (75-97\%), su baja especificidad (28\% en cuestionarios autoadministrados o del $86 \%$ cuando se cumplimentaba por personal sanitario) era origen de un inadecuado señalamiento de familias que no constituían ningún riesgo para el maltrato infantil. Por ello la USPSTF en- 
cuentra insuficiente evidencia para recomendar el rutinario cribado a padres de abuso infantil. No existen estudios que determinen la seguridad y pertinencia del uso de instrumentos de cribado en la población general para identificar familias con conductas de abuso infantil (nivel de evidencia II-2, fuerza de recomendación E).

¿Es efectiva la lista de riesgos en la consulta para identificar factores de riesgo en las familias?

Leventha ${ }^{45}$ se preocupa de está cuestión comparando 114 familias que cumplían criterios de riesgo aplicando listados de factores de riesgo frente a 114 familias de control, observando que el grupo identificado presentaba mayores frecuencias de hospitalizaciones de los niños por razones médicas o físicas, sin aclarar si se trataba de abuso infantil. No se han encontrado otras publicaciones que muestren la efectividad del cribado en la población general en las consultas de Pediatría y teniendo en cuenta que no existe un perfil familiar dominante de exposición al abuso infantil es difícil identificar correctamente las familias de riesgo de abuso infantil.

A nivel de expertos destaca que The Canadian Task Force on Preventive Health Care (CTFPHC) indica que existe buena evidencia y buena calidad para excluir procedimientos de cribado (que incluyen entrevistas o lista de riesgos) que ayuden a identificar individuos con riesgo de cometer o experimentar maltrato a niños. Recomienda la realización directamente de programas de prevención sobre familias con desventajas (madres jóvenes, sin apoyo familiar, etc.) basado en la visita a domicilio durante el periodo prenatal y durante la primera infancia para prevenir el maltrato infantil ${ }^{46}$ (nivel de evidencia II-2, fuerza de recomendación E).

Sí parece recomendable observar y recoger en la consulta datos que permitan identificar a las familias de riesgo, tales como (evidencia III, recomendación C):

- La conducta del niño.

- El comportamiento de los padres.

- La exploración clínica.

- La historia social (dinámica familiar, composición familiar, situación laboral...).

- Tiene especial importancia la observación en la consulta de:

- No acudir a las citas concertadas. - Ausencia de informes tras ingresos hospitalarios.

- Cambios frecuentes de médicos. - Acudir a consulta sin motivo aparente. 
- Imposibilidad de recabar datos de la vida familiar en la entrevista clínica.

\section{¿Se han encontrado evidencias para} recomendar actividades preventivas relativas al maltrato infantil en las familias de bajo riesgo?

No se han encontrado ensayos clínicos controlados sobre alguna de las intervenciones en la población general en el ámbito de la Atención Primaria (prevención primaria, ver tabla $\mathrm{V}$ ). Todas las publicaciones sobre prevención del maltrato infantil están referidas a intervenciones sobre poblaciones de riesgo.

No encontramos evidencias de que el aumento de habilidades de los padres o la educación en "solución de conflictos" a los padres disminuyan algún tipo de maltrato cuando se realizan en Atención Primaria de salud en poblaciones sin tener en cuenta el riesgo (nivel de evidencia III, fuerza de recomendación I).

En relación a programas de educación a la infancia para la prevención del abuso sexual anotamos dos consideraciones, la primera que es una tarea que se desarrolla en la escuela y no en los centros de salud y la segunda que existe insuficiente evidencia para recomendarla según el metaanálisis realizado por la
$\mathrm{CTFPHC}^{46}$ (nivel de evidencia I, fuerza de recomendación I).

¿Los programas multidisciplinares en familias de riesgo disminuyen los casos de abuso infantil?

Se entiende por programas multidisciplinares aquellos que engloban de manera integrada el apoyo psicológico (mejora de la autoestima, solución de conflictos, control de conductas violentas), el apoyo social con la participación de trabajadores sociales, la educación en nutrición, crianza, uso de guías anticipatorias, programas de disminución del tabaquismo, abandono de drogas o alcohol.

Brayden (1993) ${ }^{47}$ en un estudio aleatorizado casos-control de mujeres de riesgo atendidas en la etapa prenatal hasta la edad de los 2 años y seguidas durante 36 meses, no encuentra diferencias estadísticamente significativas entre el grupo que recibe apoyo psicosocial y el grupo control en cuanto a incidencia de abuso físico, curiosamente el riesgo relativo $(R R)$ de presentar maltrato negligente del grupo que recibió apoyo psicológico fue mayor ( $R R=$ 2,79; IC 95\%: 0,98-7,91, $p<0,005)$.

Wekerle $(1993)^{48}$ presenta una revisión sistemática de publicaciones desde 1977 a 1990, 34 publicaciones de ca- 
sos-control, aleatoriamente asignados. Sus hallazgos principales son que las familias de riesgo del grupo de intervención presentaban mayores puntuaciones en las áreas relativas a la crianza y puntuaciones modestas en las áreas del desarrollo cognitivo y de la conducta infantil. Estos beneficios desaparecían a largo plazo, a los 5 años. Los indicadores de disminución del maltrato eran poco fidedignos y no eran variables de estudio en la mayoría de los trabajos analizados por Wekerle.

De lo publicado podemos decir que existe insuficiente evidencia para no recomendar o recomendar esta actividad en los controles de salud (evidencia I, recomendación I).

\section{¿Los programas de educación}

para la salud en niños de riesgo de maltrato previenen este fenómeno?

Britner (1997) ${ }^{49}$ publica un estudio no aleatorizado de casos-control en el que evalúa el efecto de un programa de educación en mujeres de riesgo de 12 semanas de duración, encontrando que en el grupo de intervención disminuyeron los informes de abuso y abandono frente al grupo control.

Las dificultades para considerar esta una actividad con suficiente evidencia en Atención Primaria a partir de este trabajo provienen del diseño metodológico (no aleatorizado y escasa información de las variables de los sujetos de cada grupo), pero además de las dificultades que presenta en nuestro medio implantar una actividad educativa con tanta dedicación y en la que es necesario la preparación de los formadores o educadores.

Existe pues evidencia conflictiva para no recomendar o recomendar la educación dirigida a padres de riesgo en el programa de actividades preventivas (evidencia II-1, recomendación C).

La CTFPHC en relación a las dos preguntas anteriores concluye que es insuficiente la evidencia encontrada para recomendar programas de salud integrados con otros profesionales, la educación de padres y programas de ayuda social, incluida la psicoterapia para la prevención en el sector salud del maltrato infantil, si bien estas intervenciones pueden recomendarse por otras razones (evidencia II-1, recomendación I).

\section{¿El aumento de las visitas de seguimiento disminuye el maltrato infantil en familias de riesgo?}

En el estudio de Brayden ${ }^{47}$ el grupo de familias de alto riesgo asignado a la intervención recibió un promedio de 17,7 +/- 11,5 controles de salud frente al 
grupo control que tuvo $8,3+/-8,4$ visitas, los resultados no evidenciaron una disminución en el número de abusos o trato negligente en el grupo de intervención. Existe poca evidencia para excluir o incluir esta actividad en los controles de salud para prevenir el maltrato en la infancia (evidencia II-1, recomendación I).

\section{¿Los programas de visita a} domicilio en familias de riesgo disminuyen el maltrato infantil?

Desde la actualización en el año 2000 de la $\mathrm{CTFPHC}^{46}$ se han publicado dos revisiones sistemáticas más sobre prevención del maltrato infantil, con especial dedicación a la visita a domicilio por personal sanitario en familias de riesgo, las de la USPSTF (2004) $)^{41}$ y la Task Force on Community Preventive Services (TFCPS) $(2003)^{50}$. Las tres revisiones se han realizado con la mejor metodología aplicable a las revisiones sistemáticas y han llegado a la misma conclusión: existe buena evidencia para recomendar programas de visita a domicilio desde el periodo perinatal y durante la infancia para prevenir el abuso y abandono en familias con desventajas o riesgo (evidencia I, recomendación A).

Las tres revisiones basan sus resultados en el valor principal que le dan a los trabajos de Olds $s^{51-53}$ que analiza el impacto en la incidencia de maltrato a los 2, 4 y 15 años de la intervención de la familia en el programa de visita domiciliaria denominado The Elmira, donde se observan diferencias entre el grupo de intervención y el de control a los 2 años, reducción de estas diferencias, en términos de incidencia de abuso infantil a los 4 años y nuevamente un incremento de las diferencias a los 15 años de seguimiento (diferencias de frecuencias ajustadas de informes de abuso o abandono infantil entre el grupo control y el de intervención de 0,77 (IC 95\%: 0,34-1,19; $\mathrm{p}<0,001)$.

Estos resultados contrastan con las revisiones sistemáticas publicadas en 1996 por Roberts ${ }^{54}$ y por la librería Cochrane $^{55}$ en 1999 donde se comunican resultados más bien modestos, odds ratio de padecer abuso físico o abandono de 0,98 (IC 95\%: 0,62-1,53) o de 1,12 (IC 95\%: 0,80-1,57) respectivamente, advirtiendo ambas revisiones que las diferencias metodológicas de los artículos revisados (inapropiada aleatorización, seguimientos cortos en el tiempo a los grupos, ensayos sin doble ciego y criterios de clasificación de riesgo diferentes o diferencias en las variables de medidas) y los distintos recursos empleados en las intervenciones podrían 
estar subestimando el efecto de la visita a domicilio. Así, algunos programas de visita a domicilio estaban realizados por personal parasanitario o voluntarios. Resumen de estos programas se pueden leer en la publicación de Rubrin (2001) ${ }^{56}$.

Un ensayo clínico posterior publicado por Duggan ${ }^{57}$ no encontró diferencias en la prevención del abuso, $(O R=1,30$; IC 95\%: 0,89-1,88; $p=0,17$ ), pero sí observó un efecto modesto en la prevención del trato negligente en las familias de riesgo que participaban en el programa de visita domiciliaria $(\mathrm{OR}=$ 0,72; IC 95\%: 0,54-0,96; $p=0,02$ ).

Podemos concluir que existen suficientes evidencias para recomendar la visita domiciliaria desde la etapa prenatal hasta los dos primeros años de vida en familias de riesgo de cometer abuso infantil (evidencia I, recomendación B).

\section{¿Cuál es el tamaño del efecto}

de la visita a domicilio sobre

la prevención del abuso infantil?

En la revisión realizada por la TFCPS ${ }^{50}$ se estima que como media se espera una reducción del $40 \%$ en la incidencia del abuso en el grupo que recibe programas de visita a domicilio frente al grupo control, a corto plazo y a largo plazo, 15 años después.

Efectos a largo plazo publicados ${ }^{40-42}$ : reducción de conductas criminales de la madre; disminución del uso de las ayudas sociales; y disminución del abuso de drogas y alcohol en la madre.

\section{¿Los costes de prevención superan} a los costes de atención al maltrato

\section{infantil?}

No hemos encontrado ningún estudio relativo a costes y beneficios económicos de la prevención del maltrato infantil en España.

The National Clearinghouse on Child Abuse and Neglect Information ${ }^{58}$ publicó en su boletín de febrero del 2003 tres estudios de costes de no prevenir el abuso infantil en EE. UU.

El estudio de Elmira, New York (Olds, 1993), estima que los costes directos de los servicios sociales, policiales y sanitarios de atención al abuso excedió a los costes del programa de visita a domicilio en aproximadamente 300 dólares por familia y año (dólares de 1980).

- El segundo estudio es el de Michigan (1992), en él se refiere que los costes del programa de visita domiciliaria fue de 712 dólares por familia y año, representado un total de gastos de 42 millones de dólares por año para este programa. En contraste los gastos de atención al maltrato e inadecuada 
atención prenatal fueron de 832 millones de dólares.

El tercer estudio es el de Colorado (1995), en este trabajo se estima en 190 millones de dólares los costes directos de atención al maltrato y 212 millones los costes indirectos, lo que hacen un total de 402 millones de dólares. En contraste los gastos en prevención fueron de 2.000 dólares por familia y año, representando 8 millones de dólares al año. Estimando una reducción de solo un $6 \%$ del maltrato debido al programa de visita a domicilio, esperaríamos un ahorro de 24 millones de dólares al año realizando programas de visita domiciliaria a poblaciones de riesgo.

Es indudable que las diferencias encontradas en estos programas se deben a las diferencias en los mismos en cuanto a personal de intervención, características del propio programa y la institución que lo ampara, consideración de las partidas de costes directos e indirectos del maltrato, pero sobre todo las diferencias parten de los resultados que se desean obtener además de la disminución del abuso infantil, ya que modifican la intensidad del programa en recursos humanos y económicos (disminución del abuso, prevención del embarazo no deseado en la siguiente gestación, prevención del bajo peso al nacer, prevención del absentismo escolar, tratamiento del abuso a drogas, de los problemas de salud mental, etc.).

Aun así podemos decir que los positivos resultados de los programas de prevención, con relativamente pequeñas reducciones en la frecuencia del maltrato, son coste-efectivos.

\section{¿Qué características tiene}

el programa de visita a domicilio de familias de riesgo?

Existen programas tanto en EE. UU. como Canadá y Europa (Francia, Dinamarca o Reino Unido) y si bien pueden diferir en su intensidad (frecuencia de visitas), duración, personal que lo atiende, relación con los servicios de Atención Primaria y contenidos más o menos extensivos y multidisciplinares, la mayoría de estos programas tienen en común ${ }^{41,42:}$

1. Están dirigido a las familias con desventajas, padres jóvenes, familias monoparentales, familias de clase socioeconómica baja, familias con nacidos de muy bajo peso, padres previamente investigados por maltrato infantil y padres con abuso de alcohol, drogas, problemas de salud mental o enfermos de SIDA.

2. Dichos programas se inicia en la semana 18 de gestación y continúan has- 
ta el primer o segundo año de vida del niño.

3. Las visitas las realizan habitualmente personal de Enfermería adiestrado, en algunos casos personal parasanitario.

4. Entre los contenidos se incluyen la educación a los padres, atención a las necesidades de salud de la familia, so- porte o ayuda social y compromisos de los padres con el centro de salud y los servicios sociales.

5. Las visitas durante el embarazo son cada 1-2 semanas y posteriormente cada 2-4 semanas hasta el segundo año de vida. La duración de cada visita es de 60 a 90 minutos.

\section{Bibliografía}

1. Centro Reina Sofía para el estudio de la violencia. Maltrato infantil en la familia. Comunidad Valenciana (1997/1998). Valencia: Centro Reina Sofía; 2000.

2. Maltrato Infantil en la familia. España (1997/1998). Valencia: Centro Reina Sofía; 2002.

3. López F. Abusos sexuales a menores. Lo que recuerdan de mayores. Madrid: Ministerio de Asuntos Sociales; 1994.

4. Creighton S. Prevalence and incidence of child abuse: international comparisons. NSPCC Inform. 2004 [consultado el 13/01/2009]. Disponible en www.nspcc.org.uk/Inform/research /Briefings/prevalenceandincidenceofchildabu se_wda48217.html

5. Cerezo MA, Más E, Simó S. Análisis y evaluación de los indicadores de riesgo en situaciones de maltrato infantil. (Memoria final de proyecto). Valencia: Dirección General de Servicios Sociales, Generalitat Valenciana; 1998. p. 27, 39-53.

6. Edwards VJ, Holden GW, Felitti VJ, Anda RF. Relation between multiple forms of childhood maltreatment and adult mental health in community respondents: results from the adverse childhood experiences study. Am J Psychiatry. 2003;160:1453-60.
7. Scher $C D$, Forde $D R, M c Q u a i d ~ J R$, Stein $M B$. Prevalence and demographic correlates of childhood maltreatment in an adult community sample. Child Abuse Negl. 2004;28:167-80.

8. Gushurst CA. Chils abuse: behavioral aspects and other associated problems. Pediatr Clin N Am. 2003;50:919-38.

9. Brodsky BS, Oquendo M, Ellis SP. The relationship of childhood abuse to impulsivity and suicidial behavior in adults with mayor depression. Am J Psychiatry. 2001;158:1871-7.

10. MacMillan $\mathrm{HL}$, Fleming JE, Streiner DL. Chilhood abuse and lifetime psychopathology in a community sample. Am J Psychiatry. 2001; 158:1879-83.

11. Lansford J, Dodge KA, Pettet GS. A 12-year prospective study of the long-term effects of early child physical maltreatment of psychological, behavioural, and academic problems in adolescence. Arch Pediatr Adolesc Med. 2002; 156:824-30

12. Wolfe DA, Scott K, Wekerle C. Child maltreatment: risk of adjustment problems and dating violence in adolescence. J Am Acad Child Adolesc Psychiatry. 2001;40:282-9.

13. Osuna MJ, Cabrera JH, Morales MC. Estudio de las consecuencias del abandono físico en el desarrollo psicológico de niños de edad prees- 
colar en España. Chil Abuse Negl. 2000;24:91124.

14. Morales JM, Zunzunegui Pastor V, Martínez Salceda V. Modelos conceptuales del maltrato infantil: una aproximación biopsicosocial. Gac Sanit. 1997:11:231-41.

15. Ertem IO, Leventhal JM, Dobbs S. Intergeneracional continuity of child physical abuse. How good is the evidence? Lancet. 2000; 356:814-9.

16. McGloin JM, Widom C. Resilience among abused and neglected children grown up. Dev Psychopathol. 2001;13:1021-38.

17. Valle LA, Silovsky JF. Attibutions and adjustment following child sexual and physical abuse. Child Maltreatment. 2002;7:9-24.

18. Aracena M, Castillo R, Haz A. Resiliencia al maltrato físico infantil: variables que diferencian a los sujetos que maltratan y no maltratan físicamente a sus hijos en el presente y que tienen historia de maltrato físico en la infancia [consultado el 13/01/2009]. Disponible en www.facso.uchi le.cl/publicaciones/psicologia/docs/resiliencia_ maltrato.pdf

19. Balge KA, Milner JS. Emotion recognition ability in mothers at high and low risk for child physical abuse. Child Abuse Negl. 2000;24: 1289-98.

20. Zelenko M, Lock J, Kraemer HC, Steiner H. Perinatal complications and child abuse in a povert sample. Child Abuse Negl. 2000;24:939-50.

21. Dopke CA, Milner JS. Impact of child noncompliance on stress appraisals atributions, and disciplinary choices in mothers at high and low risk for child physicial abuse. Child Abuse Negl. 2000;24:494-504.

22. Gillham B, Tanner G, Cheyne B, Freeman I, Rooney $M$, Lambie A. Unemployment rates, single aprent density, and indices of child poverty: their relationship to different categories of child abuse and neglect. Child Abuse Negl. 1998:22:79-90.

23. Drake B, Pandey S. Understanding the relationship between neighborhood poverty and specific types of child maltreatment. Child Abuse Negl. 1996;20:1003-18.

24. Kotch JB, Browne DC, Ringwalt CL, Dufort V, Ruina E, Stewart PW, et al. Stress, social support, and substantiated maltreatment in the second and third years of life. Child Abuse Negl. 1997;21:1025-37.

25. Wilson LM, Reid AJ, Midmer DK, Biringer A, Carroll JC, Stewart DE. Antenatal psychosocial risk factors associated with adverse postpartum family outcomes. CMAJ. 1996;154:785-99.

26. Brow J, Cohen P, Johnson JG, Salzinger $S$. A longitudinal analysis of risk factors for child maltreatment: findingd of a 17-year prospective study of officially recorded and self-reported child abuse and neglect. Child Abuse Negl. 1998;22:1065-78.

27. Oliván G. Maltrato en niños con discapacidades: características y factores de riesgo. An Esp Pediatr. 2002;56:219-23.

28. Zunzunegui MV, Morales JM, Martínez V. Maltrato infantil: factores socioeconómicos y estado de salud. An Esp Pediatr. 1997;47:33-41.

29. American Academy of Pediatrics Committee on Child Abuse and Neglect. The role of the pediatrician in recognizing and intervening on behalf of abused women. Pediatrics. 1998; 101: 1091-2.

30. Hartley CC. The co-occurrence of child maltreatment and domestic violence: examining both neglect and child physical abuse. Child Maltreat. 2002;7:349-58.

31. McGuigan WM, Pratt CC. The predictive impact of domestic violence on three types of child maltreatment. Child Abuse Negl. 2001; 25:869-83. 
32. Bethea L. Primary prevention of child abuse. Am Fam Physician. 1999;59:1577-85, 1591-2.

33. Nester CB. Prevention of child abuse and neglect in the primary care setting. Nurse Pract. 1998;23:61-2, 67-70, 73.

34. Siegel R, Hill $T$, Henderson V, Ernst $H$, Boat $\mathrm{B}$. Screening for domestic violence in the community pediatric setting. Pediatrics. 1999;104: 874-7.

35. American Academy of Pediatrics Committee on Child Abuse and Neglect. The role of the pediatrician in recognizing and intervening on behalf of abused women. Pediatrics. 1998; 101:1091-2.

36. Whipple EE, Richey CA. Crossing the line from physical discipline to child abuse: how much is too much? Child Abuse Negl. 1997; 21:431-44.

37. Jackson $\mathrm{S}$, Thompson RA, Christiansen $\mathrm{EH}$, Colman RA, Wyatt J, Buckendahl CW, et al. Predicting abuse-prone parental attitudes and discipline practices in a nationally representative sample. Child Abuse Negl. 1999;23:15-29.

38. Youssef RM, Attia MS, Kamel MI. Children experiencing violence. I: Parental use of corporal punishment. Child Abuse Negl. 1998;22:959-73.

39. Fetsch RJ, Schultz CJ, Wahler JJ. A preliminary evaluation of the Colorado rethink parenting and anger. Child Abuse Negl. 1999;23:353-60.

40. American Academy of Pediatrics. Committee on Child and Adolescent Health. The role of homevisitation programs in improving health outcomes for children and families. Pediatrics. 1998;101:486-9.

41. Gomby D, Culross P, Behrman R. Home visiting: recent program evaluations. Analysis and recomendations. The Future of Children. 1999;9:4-26.

42. Olds $D$, Henderson Ch, Kitzman $H$, Eckenrode JJ, Cole R, Tatelbaum R. Prenatal and in- fancy home visitation by nurses: recent findings. The Future of Children. 1999;9:44-63.

43. Rushton FE. The role of health care in child abuse and neglect prevention. J S C Med Assoc. 1996;92:133-6.

44. Nygren $P$, Nelson $H$, Klein J. Screening children for family violence. A review of the evidence for the US Preventive Services Task Force. Ann Fam Med. 2004;2:161-9.

45. Leventhal JM, Pew MC, Berg AT, Garber $\mathrm{RB}$. Use of health services by children who were identified during the postpartum period as being at high risk of child abuse or neglect. Pediatrics. 1996;97:331-5.

46. MacMillan HL. Canadian Task Force on Preventive Health Care. Preventive health care, 2000 update: prevention of child maltreatment. CMAJ. 2000;163:1451-8.

47. Brayden RM, Altemeier WA, Dietrich MS, Tucker DD, Christensen MJ, McLaughlin FJ, et al. A prospective study of secondary prevention of child maltreatment. J Pediatr. 1993;122:511-6.

48. Wekerle C, Wolfe DA. Prevention of child physical abuse and neglect: promising new directions. Clin Psychology Rev. 1993;13:501-40.

49. Britner PA, Reppucci ND. Prevention of child maltreatment: evaluation of a parent education program for teen mothers. J Child Fam Studies. 1997;6:165-75.

50. Hahn RA, Bilukha OO, Crosby A. First reports evaluating the effectiveness of strategies for preventing violence: early childhood home visitation. Findings from the Task Force on Community preventive Services. MMWR Recomm Rep. 2003;52(RR-14):1-9.

51. Olds DL, Henderson CR Jr, Kitzman $\mathrm{H}$. Does prenatal and infancy nurse home visitation have enduring effects on qualities of parental caregiving and child health at 25 to 50 months of life? Pediatrics. 1994;93:89-98. 
52. Olds DL, Eckenrode J, Henderson CR Jr, Kitzman H, Powers J, Cole R, et al. Long-term effects of home visitation on maternal life course and child abuse and neglect: fifteen-year follow-up of a randomized trial. JAMA. 1997;278:637-43.

53. Kitzman $\mathrm{H}$, Olds DL, Henderson CR Jr, Hanks C, Cole R, Tatelbaum R, et al. Effect of prenatal and infant home visitation by nurses on pregnancy outcomes, childhood injuries, and repeated childbearing: a randomized controlled trial. JAMA. 1997;278:644-52.

54. Roberts I, Kramer MS, Suissa S. Does home visiting prevent childhood injury? A systematic review of randomised controlled trials. BMJ. 1996:312:29-33.

5. Hodnett ED, Roberts I. Home-based social support for socially disadvantaged mothers. (Cochrane Rewiew). In: The Cochrane Lybrary, Issue 2, 1999. Oxford: Update Software.

56. Rubin D, Lane W, Ludwig S. Child abuse prevention. Curr Opin Pediatr. 2001;13:388401.

57. Duggan A, McFarlane E, Fuddy L. Randomized trial of a statewide home visiting program: impact in preventing child abuse and neglect. Child Abuse Negl. 2004;28:597-622.

58. National Clearinghouse on Child Abuse and Neglect Information. Prevention pays: The cost of not preventing child abuse and neglect. Febrero 2003 [consultado el 08/02/2005]. Disponible en www.nvbest.org/childabuse/costof notprev.pdf 